\title{
Radical Resection After IORT-Containing Multimodality Treatment is the Most Important Determinant for Outcome in Patients Treated for Locally Recurrent Rectal Cancer
}

\author{
Raphaëla C. Dresen, MD, ${ }^{1}$ Marleen J. Gosens, MSc, ${ }^{1}$ Hendrik Martijn, MD, PhD ${ }^{2}$ \\ Grard A. Nieuwenhuijzen, MD, PhD, ${ }^{1}$ Geert-Jan Creemers, MD, PhD, ${ }^{3}$ \\ Alette W. Daniels-Gooszen, MD, PhD, ${ }^{4}$ Adriaan J. van den Brule, MSc, $\mathrm{PhD},{ }^{5}$ \\ Hetty A. van den Berg, MD, ${ }^{2}$ and Harm J. Rutten, MD, $\mathrm{PhD}^{1}$
}

\author{
${ }^{1}$ Department of Surgery, Catharina Hospital Eindhoven, Postbox 1350, 5602 ZA Eindhoven, The Netherlands \\ ${ }^{2}$ Department of Radiotherapy, Catharina Hospital, Eindhoven, The Netherlands \\ ${ }^{3}$ Department of Oncology, Catharina Hospital, Eindhoven, The Netherlands \\ ${ }^{4}$ Department of Radiology, Catharina Hospital, Eindhoven, The Netherlands \\ ${ }^{5}$ Laboratory for Pathology, PAMM Laboratories, Eindhoven, The Netherlands
}

\begin{abstract}
Background: The optimal treatment for locally recurrent rectal cancer (LRRC) is still a matter of debate. This study assessed the outcome of LRRC patients treated with multimodality treatment, consisting of neoadjuvant radio (chemo-) therapy, extended resection, and intraoperative radiotherapy.

Methods: One hundred and forty-seven consecutive patients with LRRC who underwent treatment between 1994 and 2006 were studied. The prognostic values of patient-, tumor- and treatment-related characteristics were tested with uni- and multivariate analysis.

Results: Median overall survival was 28 months (range 0-146 months). Five-year overall, disease-free, and metastasis-free survival and local control (OS, DFS, MFS, and LC respectively) were $31.5 \%, 34.1 \%, 49.5 \%$ and $54.1 \%$ respectively. Radical resection (R0) was obtained in 84 patients $(57.2 \%)$, microscopically irradical resection (R1) in 34 patients $(23.1 \%)$, and macroscopically irradical resection (R2) in 29 patients $(19.7 \%)$. For patients with a radical resection median OS was 59 months and the 5-year OS, DFS, MFS, and LC were $48.4 \%$, $52.3 \%, 65.5 \%$ and $68.9 \%$, respectively. Radical resection was significantly correlated with improved OS, DFS, and LC $(P<0.001)$. Patients who received re-irradiation or full-course radiotherapy survived significantly longer $(P=0.043)$ and longer without local recurrence $(P=0.038)$ or metastasis $(P<0.001)$ compared to patients who were not re-irradiated.

Conclusions: Radical resection is the most significant predictor of improved survival in patients with LRRC. Neoadjuvant radio (chemo-) therapy is the best option in order to realize a radical resection. Re-irradiation is feasible in patients who already received irradiation as part of the primary rectal cancer treatment.
\end{abstract}

Key Words: Rectal cancer-Local recurrence-Multimodality treatment-IORT-Re-irradiation.

Published online April 4, 2008.

Address correspondence and reprint requests to: Harm J. Rutten, MD, PhD; E-mail: harm.rutten@cze.nl

Published by Springer Science+Business Media, LLC $\odot 2008$ The Society of Surgical Oncology, Inc.
The introduction of total mesorectal excision (TME) surgery and neoadjuvant radio (chemo-) therapy (RCT) has resulted in a decrease of the incidence of locally recurrent rectal cancer (LRRC) to below $10 \%{ }^{1-6}$ However, patients who do develop 
LRRC still have limited treatment options and subsequent prognosis. Due to prior surgery, tumor growth is not confined to a specific compartment lined by fascias, because these fascias have been damaged during the primary surgery. Therefore recurrences easily grow into surrounding compartments. Radicality can often only be achieved by a multicompartmental resection. These resections are accompanied by high morbidity and mortality rates up to $10 \% .^{7-9}$ Neoadjuvant treatment options are often limited because the patients have already received radio (chemo-) therapy during the treatment of the primary tumor. Tissue tolerance for re-irradiation is limited to approximately 30 Gray (Gy) due to dose accumulation toxicity. ${ }^{10}$ Furthermore, it can be hypothesized that a recurrent tumor shows a more aggressive biological behavior. It has been demonstrated that more than $50 \%$ of patients with LRRC will develop a metastasis within 6 months after the diagnosis of the local recurrence. ${ }^{11}$

The inherent poor prognosis often leads to a nihilistic approach (with a high tumor-related morbidity), depriving patients of a chance of cure. However, curative treatment has improved slightly in specialized centers over the years, due to the use of neoadjuvant RCT, radical resection and in some centers intraoperative radiotherapy (IORT). IORT, either as electron-beam therapy (IOERT) or as brachytherapy, enables accurate delivery of a radiation dose to the tumor bed. Dose limitations of normal tissue can be minimized either by temporary removal out of the irradiation volume or by lead shielding. The use of external beam irradiation (EBRT) in combination with IORT allows local delivery of a tumorcidal biological dose up to 80 $90 \mathrm{~Gy} .{ }^{12,13}$

As LRRC patients constitute a highly heterogeneous group, often presenting with concomitant metastatic disease, and randomized studies are difficult to perform. Most of the knowledge about the treatment of LRRC patients is empirical and obtained from centers able to accumulate sufficient data. Since 1994, the Catharina Hospital has been a national referral center for patients with LRRC in the Netherlands.

This retrospective study of a large patient population assessed whether intensive multimodality treatment, consisting of neoadjuvant radiotherapy (often combined with chemotherapy), extended resection, and IORT was useful in locally recurrent rectal cancer patients. Furthermore, the role of re-irradiation was determined.

\section{PATIENTS AND METHODS}

\section{Patients}

From March 1994 to July 2006, 184 consecutive patients with LRRC within the pelvis, without identified distant metastases at time of diagnosis, were scheduled for treatment with curative intent. For most patients this treatment comprised multimodality treatment, but some patients in early years did not receive neoadjuvant treatment. Multimodality treatment consisted of neoadjuvant radio (chemo-) therapy, extended surgery, and IOERT. Patients were referred to our institution from 42 hospitals throughout the Netherlands. Upon referral, all data about the primary and recurrent rectal tumor were available. Thirty-seven patients were excluded from further analysis because of the following reasons: 27 patients proved to have irresectable disease because of tumor growth into S2 or higher $(n=20)$, intraperitoneal metastases $(n=10)$, and infiltration into the neural plexus, which extended beyond the infrapiriform foramen $(n=2)$. Ten patients were excluded because they had a history of metastasectomy. The remaining 147 patients form the basis of this study. Patients' charts, operation, and pathology reports were reviewed in order to obtain patient demographics and treatment history. After resection of the LRRC, most patients returned to their initial hospital for follow-up. Follow-up data could be completed in all patients either by medical record review or by contacting patients or their treating physicians by phone. Median followup time for survivors was 34.0 months (range 6146 months). Our institutional review committee approved this analysis and waived informed consent, because of the retrospective nature of the study.

\section{Preoperative Evaluation}

The local recurrences were discovered by routine follow-up or symptoms. In most patients histological confirmation could be obtained by either direct or computed tomography (CT)-guided biopsy. Progressive growth on repeated magnetic resonance imaging (MRI) or CT scans, especially in combination with increased carcino-embryonic antigen level or positive positron emission tomography (PET), was sometimes considered as sufficient evidence for the diagnosis of a local recurrence. All patients received a CT and/or a MRI of the pelvis in order to assess the localization of the recurrence and the extent of growth into surrounding structures. In order to exclude disseminated 
disease patients underwent a liver ultrasound and a chest X-ray and since the year 2000 a CT scan of the chest and abdomen, in difficult cases supplemented with a PET scan.

\section{Neoadjuvant Therapy}

Neoadjuvant treatment regimen has changed over the years, because of changes in treatment protocol. In the early years (1994-1999), patients received preoperative radiotherapy alone as external beam radiation therapy (EBRT) with a dose of 50.4 Gray (Gy), five times a week in 28 doses of $1.8 \mathrm{~Gy}$. If the patient had already received radiotherapy for the primary tumor, no re-irradiation was given to the recurrent tumor. In 1997, re-irradiation (30.6 Gray, five times a week in 17 doses of $1.8 \mathrm{~Gy}$ ) was introduced in previously irradiated patients. EBRT was delivered with a linear accelerator $(10 \mathrm{MeV})$ by using a three-field technique, utilizing one posterior and two lateral portals. The pelvic field borders were: the lateral borders extending $1-1.5 \mathrm{~cm}$ lateral of the bony pelvis, the cranial borders being the promontory (L5S1), the caudal border was below the obturator foramen. The dorsal border encompassed the sacrum and the anterior border was chosen in such a way that the initial tumor region was widely covered. If the local recurrence was low seated, the perineum was included in the radiation volume. If necessary, a spacer was placed and a stoma was constructed. The spacer was placed to displace the small bowel out of the pelvis as much as possible. A biological spacer like the omentum or the distal sigmoid was preferred and if not feasible an artificial spacer (mamma prosthesis) was used instead.

Since 1999, patients received neoadjuvant radiochemotherapy instead of radiotherapy alone. From 1999 until 2004, this consisted of 5-FU $\left(350 \mathrm{mg} / \mathrm{m}^{2} /\right.$ d) combined with leucovorin $\left(20 \mathrm{mg} / \mathrm{m}^{2} / \mathrm{d}\right)$ during the first 5 days of re-irradiation or during the first and last 5 days of full course radiotherapy. Since 2004, patients received either capecitabine $\left(2 \times 825 \mathrm{mg} / \mathrm{m}^{2} /\right.$ d) 5 days a week for three weeks combined with oxaliplatin $\left(50 \mathrm{mg} / \mathrm{m}^{2}\right)$ on day 1,8 , and 15 or continuous capecitabine $\left(2 \times 825 \mathrm{mg} / \mathrm{m}^{2} / \mathrm{d}\right) 5$ days a week alone. No adjuvant chemotherapy was administered, as this was not part of treatment guidelines in the Netherlands.

\section{Surgery}

Surgery was planned 8-10 weeks after completion of EBRT. At the start of the laparotomy, the abdo-
TABLE 1. Patient and treatment characteristics

\begin{tabular}{|c|c|}
\hline & $\begin{array}{l}\text { No. of patients } \\
\quad(n=147)\end{array}$ \\
\hline Median age, years (range) & $62(39-87)$ \\
\hline \multicolumn{2}{|l|}{ Gender } \\
\hline Male & $84(57.1)$ \\
\hline Female & $63(42.9)$ \\
\hline \multicolumn{2}{|l|}{ Stage of primary tumor } \\
\hline I & 17 (11.6) \\
\hline II & $68(46.3)$ \\
\hline III & $60(40.8)$ \\
\hline Unknown & $2(1.3)$ \\
\hline \multicolumn{2}{|l|}{ Number of LR } \\
\hline First LR & $133(90.5)$ \\
\hline Second LR & $11(7.5)$ \\
\hline Third LR & $3(2.0)$ \\
\hline $\begin{array}{l}\text { Median interval primary-recurrent } \\
\text { surgery in months (range) }\end{array}$ & $28(3-207)$ \\
\hline \multicolumn{2}{|l|}{ Type of primary surgery } \\
\hline Low anterior resection & $80(54.4)$ \\
\hline Abdominoperineal resection & $44(30.0)$ \\
\hline Rectosigmoid resection & $18(12.2)$ \\
\hline Exenteration & $2(1.4)$ \\
\hline Local procedure & $3(2.0)$ \\
\hline \multicolumn{2}{|l|}{ Type of LRRC surgery } \\
\hline Low anterior resection & $26(17.7)$ \\
\hline Abdominoperineal resection & $48(32.7)$ \\
\hline Abdominotranssacral resection & $39(26.5)$ \\
\hline Pelvic exenteration & $17(11.6)$ \\
\hline Nonanatomic & 17 (11.6) \\
\hline \multicolumn{2}{|l|}{ EBRT for primary tumor } \\
\hline \multicolumn{2}{|l|}{ No } \\
\hline -EBRT for LRRC (FC) & $66(44.9)$ \\
\hline -no EBRT for LRRC & $3(2.0)$ \\
\hline \multicolumn{2}{|l|}{ Yes } \\
\hline -EBRT for LRRC (RI) & $57(38.8)$ \\
\hline -no EBRT for LRRC & $21(14.3)$ \\
\hline \multicolumn{2}{|l|}{ Neoadjuvant treatment for LRRC } \\
\hline No & $24(16.3)$ \\
\hline RI without chemo & $13(8.9)$ \\
\hline RI with chemo & $44(29.9)$ \\
\hline FC without chemo & $23(15.6)$ \\
\hline FC with chemo & $43(29.3)$ \\
\hline \multicolumn{2}{|l|}{ Dose IOERT (Gy) } \\
\hline 0 & $11(7.5)$ \\
\hline 10 & $72(49.0)$ \\
\hline 12.5 & $35(23.8)$ \\
\hline 15 & $18(12.2)$ \\
\hline 17.5 & $11(7.5)$ \\
\hline \multicolumn{2}{|l|}{ Radicality of resection } \\
\hline R0 & $84(57.2)$ \\
\hline $\mathrm{R} 1$ & $34(23.1)$ \\
\hline R2 & $29(19.7)$ \\
\hline
\end{tabular}

Values in parentheses are percentages unless indicated otherwise. LR, local recurrence; LRRC, locally recurrent rectal cancer; EBRT, external beam radiation therapy; Gy, Gray; RI, re-irradiation; FC, full-course radiotherapy.

men was carefully checked for liver, nodal, or peritoneal metastases. In all patients a radical resection was intended. In order to obtain this, resection with extended circumferential margins was necessary. ${ }^{14}$ The different surgical procedures are described in Table 1. Radicality of resection was defined as follows: a R0 resection had free surgical margins, a R1 
resection had focally microscopically involved margins, and a R2 was defined as more than $1 \mathrm{~cm}^{2}$ involved margins, in most cases macroscopically visible tumor was left behind. As specimens of locally recurrent cases had no clearly defined anatomical borders, any free margin was considered as $\mathrm{R} 0$.

\section{Intraoperative Radiotherapy}

From 1994 until 2004, IOERT was given in a dedicated radiotherapy suite. In 2004-2005, a mobile unit was used in the operating theatre (Mobetron, Intraop). Since 2006 IOERT was delivered in a dedicated operating room using a fixed accelerator; an Elektra SL-25 linear accelerator with an in-housedeveloped fixed applicator system. The accuracy of dose delivery with this system is within the range of $\pm 5 \%$. The applicator diameter was chosen in such a way that it covered the area at risk within a margin of $\pm 1 \mathrm{~cm}$. If necessary, bolus material was used to ensure sufficient surface dose at the area at risk for possible residual tumor. The outcome of frozen sections taken during surgery determined IOERT dose and target. When negative, 10 Gy was administered, when positive with microscopic residual disease 12.5 Gy, with macroscopic residual disease $\left(<2 \mathrm{~cm}^{2}\right)$ $15 \mathrm{~Gy}$ was applied, and when positive with gross residual disease $\left(>2 \mathrm{~cm}^{2}\right), 17.5 \mathrm{~Gy}$ was delivered. The IOERT dose was specified on the $90 \%$ isodose along the central beam axis. The depth of potential tumor involvement of the area at risk was estimated to select the energy needed to obtain an isodose of $90 \%$ $\pm 1 \mathrm{~cm}$ beyond this tumor involvement. The energy being used ranged from 6 to $18 \mathrm{MeV}$, corresponding to an isodose depth of $1.4-2.8 \mathrm{~cm}$, depending on the diameter of the applicator $(5-9 \mathrm{~cm})$, the bevel end of the applicator $\left(0-30^{\circ}\right)$, the need of bolus material, and the angle between applicator and surface to be irradiated. Eleven patients did not receive IOERT: six patients had excessive blood loss, in three patients no area at risk could be identified, one patient already received IOERT as part of the primary treatment, and in one patient the risk for morbidity was too high.

\section{Statistical Analysis}

Survival time was calculated from the LRRC resection date until the last follow-up attendance or until death. Time to recurrence, either local (within the pelvis) or distant, was calculated from the LRRC resection date until the histological or evident radiological presence of a local or distant recurrence. Overall, cancer-specific, disease-free, and metastasis-free survival, and local control (OS, CSS, DFS, MFS, and LC, respectively) curves were constructed using the Kaplan-Meier method. Differences in observed survival between groups were tested for statistical significance using log-rank testing. Factors of which the $P$-value was less than 0.10 in the univariate analysis were included in the multivariate analysis. Multivariate analysis was performed using the Cox proportional hazards regression model. Relations between various parameters were analyzed using the $\chi^{2}$ method. A difference was considered statistically significant if $P \leq 0.05$ (two-sided). Statistical analyses were performed using the SPSS statistical software program (SPSS $^{\circledR}$ for Windows Release 15.0, SPSS Inc, Chicago, IL, USA).

\section{RESULTS}

\section{Patient and Treatment Characteristics}

Details of the patients and treatment are listed in Table 1. Median anaesthesia time, including time for IOERT, was $375 \mathrm{~min}$ (range 170-660 min). Median blood loss was $4750 \mathrm{ml}$ (range 420$34,000 \mathrm{ml}$ ). Median hospital stay was 19 days (range 2-105 days). Mortality rate within 1 month after operation was $4.8 \%(n=7)$, and within 3 months it was $8.2 \%(n=12)$. During hospital admission, 95 complications were observed, in 86 patients $(58.5 \%$, Table 2). Twenty-nine patients experienced urinary retention, requiring prolonged catheterization. Another common complication was a pelvic abscess $(n=22)$, needing a re-intervention in the majority of patients. For the long-term complications, standardized questionnaires were regularly sent to the surviving patients (EORTC QLQ C 30 and, CR 38, and a more specific postoperative surgical morbidity questionnaire of our own design). We found that patients experienced long-term complications (Table 2) to some extent but, with the increase of time since the operation, the complaints decreased. We did not find any differences with regard to morbidity for the various IORT doses. However, the number of the higher doses was too low for a statistical analysis.

\section{Univariate Analysis}

Median overall survival was 28 months (range 0146 months). Actuarial 5-year OS, CSS, DFS, MFS, 
TABLE 2. Postoperative complications by type of neoadjuvant therapy

\begin{tabular}{|c|c|c|c|c|}
\hline & Total $(n=149)$ & No $(n=24)$ & Re-irradiation $(n=57)$ & Full course $(n=66)$ \\
\hline \multicolumn{5}{|l|}{ Postoperative } \\
\hline No & 61 & 9 & 22 & 30 \\
\hline Urinary retention & 29 & 4 & 13 & 12 \\
\hline Abscess & 22 & 2 & 11 & 9 \\
\hline Wound infections & 17 & 2 & 5 & 10 \\
\hline Sepsis & 6 & 0 & 4 & 2 \\
\hline Urinary tract infection & 5 & 0 & 2 & 3 \\
\hline Ileus & 4 & 2 & 1 & 1 \\
\hline Haemorrhage & 3 & 0 & 2 & 1 \\
\hline Venous embolus & 3 & 2 & 1 & 0 \\
\hline Fistula & 2 & 1 & 0 & 1 \\
\hline Urinary leakage & 2 & 1 & 1 & 0 \\
\hline Pneumonia & 2 & 0 & 1 & 1 \\
\hline \multicolumn{5}{|l|}{ Late postoperative } \\
\hline Lower extremity neuropathy & 32 & 4 & 12 & 16 \\
\hline Ureter stenosis & 8 & 0 & 4 & 4 \\
\hline 3-month mortality & 12 & 2 & 4 & 6 \\
\hline
\end{tabular}

Values are absolute numbers.

and $\mathrm{LC}$ were $31.5 \%, 38.6 \%, 34.1 \%, 49.5 \%$, and $54.1 \%$ respectively. For patients with a R0 resection, median OS was 59 months and actuarial 5-year OS, CSS, DFS, MFS, and LC were $48.4 \%, 57.5 \%$, $52.3 \%, 65.5 \%$, and $68.9 \%$, respectively.

Patient-, primary tumor treatment-, and recurrence treatment-related factors were analyzed in relation to 3-year overall survival, local control, and metastasis-free survival (Table 3). Radical resection was correlated with significantly better OS (Fig. 1), CSS, LC (Fig. 2), and MFS (Fig. 3) (all $P<0.001)$. Patients who received re-irradiation or full-course radiotherapy as part of the treatment of LRRC survived significantly longer $(P=0.043)$ and longer without local recurrence $(P=0.038)$ or metastasis $(P<0.001)$ compared to patients who were not re-irradiated. Patients who had neoadjuvant radio (chemo-) therapy experienced fewer metastases $(P<0.001)$, but did not survive longer $(P=0.170)$ than patients who did not receive neoadjuvant treatment. Early tumor-node-metastasis (TNM) stage was associated with increased OS and MFS rates $(P=0.008$ and $P=0.023$, respectively). If patients previously underwent an anterior resection, they survived 20 months longer without metastasis $(P=0.007)$ than patients who underwent an abdominoperineal resection (APR) as primary surgery, but no statistical difference was found in $\operatorname{OS}(P=0.107)$ or LC $(P=0.069)$. Patients who could be treated with LAR for the local recurrence survived 1.5 times longer than patients in whom a more extensive resection was required $(P=0.013)$.

\section{Multivariable Analysis}

The most important factors influencing overall survival were radicality of resection and stage of the primary tumor (Table 4). After irradical resection the hazard ratio (HR) for early death was 2.11 (CI 1.37$3.24, P=0.001)$. In patients with a stage II or III primary rectal cancer the mortality risk was about three times higher than in patients with stage I disease ( $P=0.014$ and $P=0.008$, respectively). Patients who had to undergo a more extensive resection for LRRC had a significantly worse prognosis (HR 2.11, CI 1.09-4.08, $P=0.027$ ) than patients only needing a LAR. No significant survival difference was found for neoadjuvant radiotherapy. In the multivariable analysis for local control, radical resection was the only significant factor $(P=0.001)$. The results of the multivariable analysis for metastasis-free survival are displayed in Table 5.

\section{Factors Influencing Radicality of Resection (Table 6)}

As our results showed radicality of resection to be the most important predictor for survival, we decided to analyze which factors were of influence on the achievement of a radical resection. Neoadjuvant treatment increased the radical resection rate; in patients who received radiotherapy (either full course or re-irradiation) or radiochemotherapy significantly more radical resections were performed than in patients who did not. After previous anterior resection, the achievement of a radical resection of the LRRC was $20 \%$ higher than after previous abdominoperineal resection $(P=0.044)$. No pain at time of 
TABLE 3. Univariate analysis of patient-, tumor-, and treatment-related factors

\begin{tabular}{|c|c|c|c|c|c|c|c|}
\hline & No. & OS at $3 y^{*}$ & $P^{\dagger}$ & LC at $3 y^{*}$ & $P^{\dagger}$ & MFS at $3 y^{*}$ & $P^{\dagger}$ \\
\hline All patients & $147(100)$ & 43.8 & & 57.0 & & 53.1 & \\
\hline \multicolumn{8}{|l|}{ Age (years) } \\
\hline$<60$ & $55(37.4)$ & 52.3 & \multirow[t]{2}{*}{0.195} & 54.1 & \multirow[t]{2}{*}{0.724} & 58.6 & \multirow[t]{2}{*}{0.525} \\
\hline$\geq 60$ & $92(62.6)$ & 38.0 & & 58.9 & & 49.6 & \\
\hline \multicolumn{8}{|l|}{ Gender } \\
\hline Male & $84(57.1)$ & 46.5 & \multirow[t]{2}{*}{0.822} & 56.7 & \multirow[t]{2}{*}{0.636} & 54.8 & \multirow[t]{2}{*}{0.332} \\
\hline Female & $63(42.9)$ & 40.3 & & 59.4 & & 51.3 & \\
\hline \multicolumn{8}{|l|}{ Stage of primary tumor } \\
\hline I & 17 (11.6) & 80.1 & \multirow{4}{*}{0.008} & 57.7 & \multirow{4}{*}{0.599} & 85.7 & \multirow{4}{*}{0.023} \\
\hline II & $68(46.3)$ & 46.9 & & 54.2 & & & \\
\hline III & $60(40.8)$ & 29.8 & & 58.3 & & 54.3 & \\
\hline Unknown & $2(1.3)$ & & & & & 39.4 & \\
\hline \multicolumn{8}{|l|}{ Type of primary surgery } \\
\hline $\mathrm{AR}$ & $98(66.7)$ & 46.0 & \multirow[t]{2}{*}{0.107} & 64.3 & \multirow[t]{2}{*}{0.069} & 58.4 & \multirow[t]{2}{*}{0.007} \\
\hline APR & $44(30.0)$ & 37.9 & & 40.7 & & 38.9 & \\
\hline \multicolumn{8}{|l|}{ Interval } \\
\hline$\leq 2$ years & $68(46.3)$ & 48.7 & \multirow[t]{2}{*}{0.728} & 57.9 & \multirow{2}{*}{0.917} & 60.6 & \multirow[t]{2}{*}{0.221} \\
\hline$>2$ years & $79(53.7)$ & 40.1 & & 55.9 & & 47.8 & \\
\hline \multicolumn{8}{|l|}{ Symptoms at diagnosis } \\
\hline So & $40(27.2)$ & 49.9 & \multirow{4}{*}{0.275} & 59.6 & \multirow[t]{4}{*}{0.031} & 55.0 & \multirow[t]{4}{*}{0.363} \\
\hline $\mathrm{S} 1$ & $47(32.0)$ & & & 74.7 & & 58.8 & \\
\hline $\mathrm{S} 2$ & $58(39.5)$ & 47.7 & & 43.3 & & 46.1 & \\
\hline Unknown & $2(1.3)$ & 36.8 & & & & & \\
\hline \multicolumn{8}{|c|}{ Dose EBRT for LRRC (Gy) } \\
\hline 0 & $24(16.3)$ & 25.0 & 0.043 & 37.6 & 0.038 & 17.8 & $<0.001$ \\
\hline 30.6 (re-irradiation) & $57(38.8)$ & 47.6 & & 48.9 & & 58.7 & \\
\hline $45-50$ (full course) & $66(44.9)$ & 49.3 & & 69.0 & & 61.2 & \\
\hline Neoadjuvant RCT for & & & & & & & \\
\hline No & $24(16.3)$ & 25.0 & 0.170 & 37.6 & 0.119 & 17.8 & $<0.001$ \\
\hline RI without chemo & $13(8.9)$ & 53.8 & & 53.3 & & 60.0 & \\
\hline RI with chemo & $44(29.9)$ & 44.9 & & 46.9 & & 54.8 & \\
\hline FC without chemo & $23(15.6)$ & 52.2 & & 69.0 & & 58.8 & \\
\hline FC with chemo & $43(29.3)$ & 47.5 & & 69.1 & & 64.2 & \\
\hline Type of LRRC surgery & & & & & & & \\
\hline LAR & $26(17.7)$ & 65.1 & 0.013 & 80.4 & 0.055 & 66.2 & 0.175 \\
\hline More extensive & $121(82.3)$ & 39.4 & & 51.4 & & 50.0 & \\
\hline Radicality of resection & & & & & & & \\
\hline R0 & $84(57.2)$ & 58.7 & $<0.001$ & 74.9 & $<0.001$ & 71.6 & $<0.001$ \\
\hline $\mathrm{R} 1$ & $34(23.1)$ & 26.5 & & 29.2 & & 30.6 & \\
\hline $\mathrm{R} 2$ & $29(19.7)$ & 24.1 & & 28.5 & & 18.8 & \\
\hline
\end{tabular}

Values in parentheses are percentages; *values are percentages of patients at three years after the operation. ${ }^{\dagger} \log$ rank test. OS, overall survival; LC, local control; MFS, metastasis-free survival; AR, anterior resection; APR, abdominoperineal resection; S0, asymptomatic; S1, symptomatic without pain; S2, symptomatic with pain; EBRT, external beam radiation therapy; LRRC, locally recurrent rectal cancer; Gy, Gray; RCT, radio(chemo-) therapy; RI, re-irradiation; FC, full-course radiotherapy; LAR, low anterior resection; R0, microscopically radical; R1 microscopically irradical; R2, macroscopically irradical.

diagnosis was also correlated with a higher rate of resections with a negative margin $(P=0.030)$.

\section{Patterns of Failure}

Median time to local recurrence was 13 months (range 1-87 months) and to metastasis 11 months (range 1-86 months). Thirty patients $(20.4 \%)$ developed metastases within 1 year, and $22(15.0 \%)$ had a local recurrence within 1 year. In the long term, around $50 \%$ developed a local recurrence or a metastasis (Figs. 2 and 3). Twenty-three patients survived more than 5 years (median 82 months; range 64-146 months). Of these patients, 7 died of the disease, 2 died of another cause, and 14 are currently alive with no evidence of disease.

\section{DISCUSSION}

This retrospective study of a large patient population assessed whether intensive multimodality treatment was useful in locally recurrent rectal cancer patients. Furthermore, the role of re-irradiation was determined. Multimodality treatment for LRRC has been described as the most encouraging method to improve survival and local control in selected patients. ${ }^{15,16}$ In the present study, an actuarial 5-year 


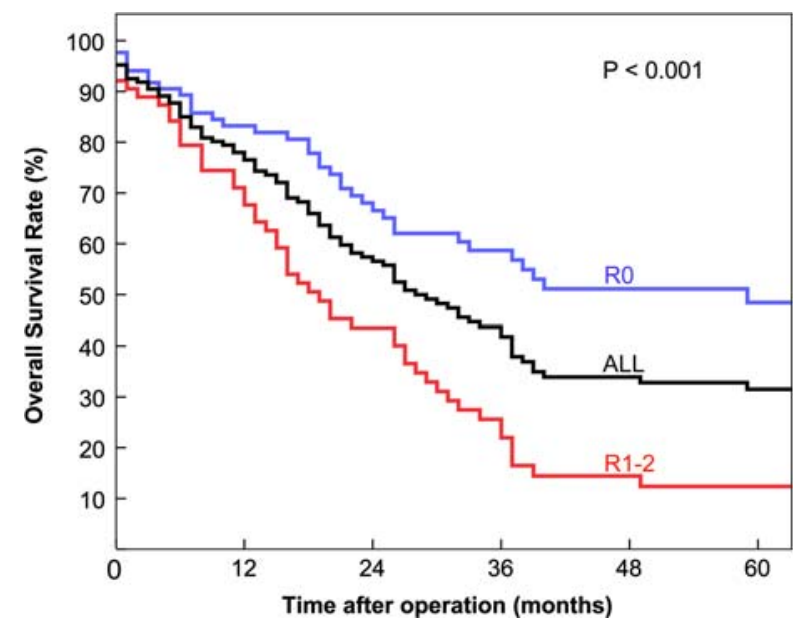

FIG. 1. Overall survival (OS) analysis. ALL, all patients $(n=147)$; R0, radically resected patients $(n=84)$; R1-R2, irradically resected patients $(n=63)$. R0 patients had a significantly better OS than R1-R2 patients $(P<0.001)$.

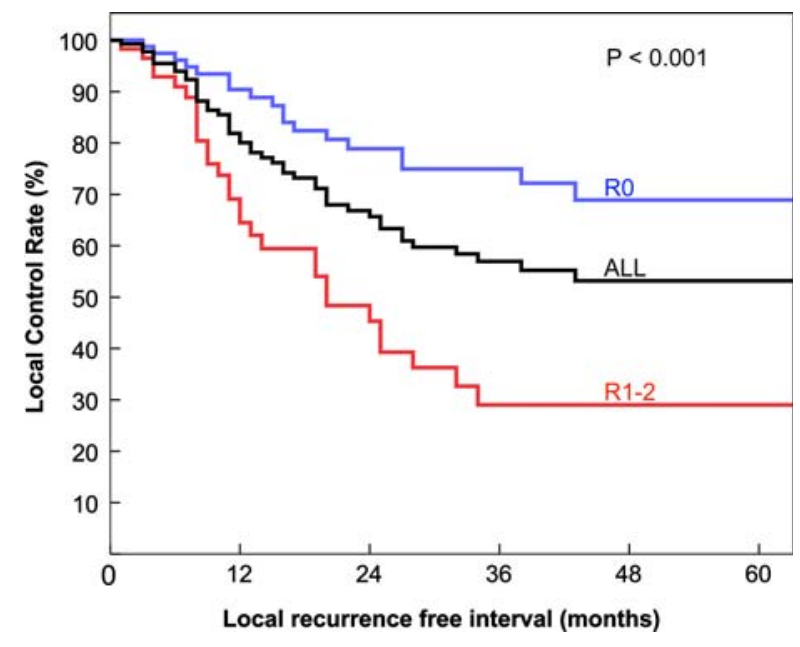

FIG. 2. Local control (LC) analysis. ALL, all patients ( $n=147)$; $\mathrm{R} 0$, radically resected patients $(n=84)$; $\mathrm{R} 1-\mathrm{R} 2$, irradically resected patients $(n=63)$. R0 patients had a significantly better LC than $\mathrm{R} 1-\mathrm{R} 2$ patients $(P<0.001)$.

survival rate of $31.5 \%$ was achieved. This outcome compares favorably to historical reports in which patients were treated with surgery alone $(24 \%)^{7}$ or with palliative treatment $(0 \%),{ }^{17}$ but still remains somewhat disappointing.

Several factors influencing the outcome of patients with locally recurrent rectal cancer were identified. The most important prognostic factor was radicality of resection. In many studies with fewer patients the importance of a radical resection was already reported. ${ }^{16,18-22}$ In this study, in $57.2 \%$ of patients a radical (R0) resection was accomplished. This percentage is consistent with the proportion of circum-

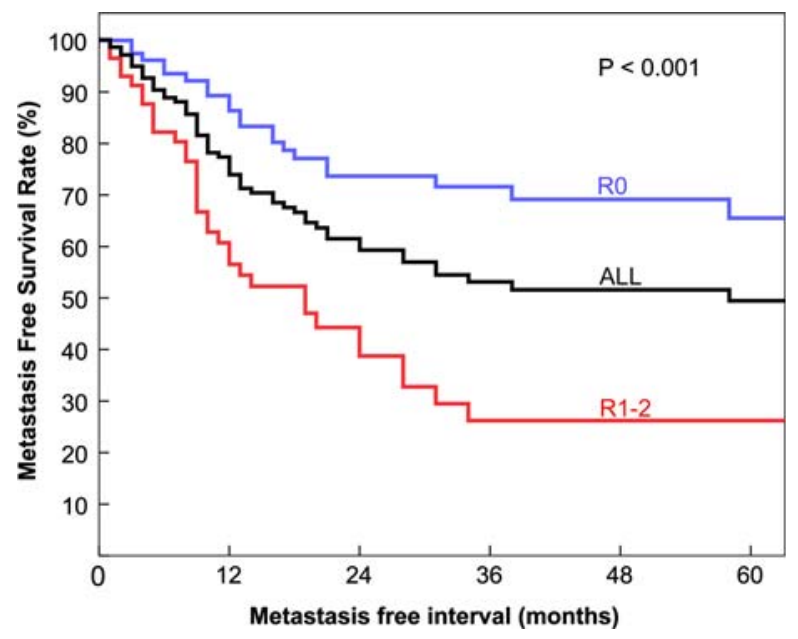

FIG. 3. Metastasis-free survival (MFS) analysis. ALL, all patients $(n=147)$; R0, radically resected patients $(n=84)$; R1-R2, irradically resected patients $(n=63)$. R0 patients had a significantly better MFS than R1-R2 patients $(P<0.001)$.

ferential margin-free resections reported in the literature. $^{21,23-27}$ Median overall survival (OS) and cancer-specific survival (CSS) after R0 in this study were 59 and 76 months, respectively. This compares favorably to previous studies, which report survival ranging from 17 to 50 months..$^{7,922,28-31}$ Unlike the finding of other authors ${ }^{32}$ that a difference may be found after gross or microscopic residual disease we did not find a difference in oncological outcome after $\mathrm{R} 1$ or $\mathrm{R} 2$ resection (Table 2). The major variable was a tumor-free margin. This may be the result of the fact that even minor residual disease represents an aggressive tumor type in our series after intensive neoadjuvant treatment. Another explanation may be the difference in staging. In our R0 group more patients with close margins were present, leaving worse patients in the R1 group.

Another important factor with a positive influence on the outcome was re-irradiation. After re-irradiation significantly more radical resection could be performed (64.9 versus $29.2 \%, P=0.004)$ and a significantly better metastasis-free survival (MFS) (at 3 years: 58.7 versus $17.8 \%, P<0.001$ ) was observed compared to surgery alone in patients who had been irradiated for their primary tumor. Other studies report a lower rate of $\mathrm{R} 0$ resections when using surgery alone. ${ }^{33-35}$ These findings stress the need for renewed radiotherapy in patients who have previously been irradiated. Our data suggest that re-irradiation even in combination with chemotherapy and intraoperative irradiation boost can be applied safely and may result in a considerable survival benefit and even cure rate. The major concern of re-irradiation is 
TABLE 4. Multivariable analysis for overall survival

\begin{tabular}{llc}
\hline & Hazard ratio & $P$ \\
\hline Radicality of resection & & \\
$\quad$ R0 & 1 & 0.001 \\
$\quad$ R1 + R2 & $2.11(1.37-3.24)$ & \\
Stage of primary tumor & 1 & 0.014 \\
$\quad$ I & $3.00(1.25-7.19)$ & 0.008 \\
$\quad$ II & $3.28(1.37-7.88)$ & \\
III & $1.17(0.67-2.03)$ & 0.588 \\
Dose EBRT for LRRC (Gy) & $0.94(0.58-1.54)$ & 0.818 \\
$\quad$ 30.6 (RI) & 1 & \\
$\quad$ 45-50 (FC) & 1 & \\
Type of LRRC surgery & $2.11(1.09-4.08)$ & 0.027 \\
$\quad$ LAR & \\
$\quad$ More extensive &
\end{tabular}

Values in parentheses are $95 \%$ confidence intervals. R0, microscopically radical; R1 microscopically irradical; R2, macroscopically irradical; EBRT, external beam radiation therapy; LRRC, locally recurrent rectal cancer; Gy, Gray; RI, re-irradiation; FC, full-course radiotherapy; LAR, low anterior resection.

TABLE 5. Multivariable analysis for metastasis-free survival

\begin{tabular}{llc}
\hline & \multicolumn{1}{c}{ Hazard ratio } & $P$ \\
\hline Radicality of resection & & \\
$\quad$ R0 & 1 & 0.018 \\
R1 + R2 & $2.05(1.13-3.72)$ & \\
Stage of primary tumor & 1 & 0.091 \\
$\quad$ I & $2.87(0.85-9.77)$ & 0.050 \\
II & $3.41(1.00-11.67)$ & \\
III & $2.24(1.14-4.39)$ & 0.019 \\
Dose EBRT for LRRC (Gy) & \\
$\quad$ 0 & $1.01(0.52-1.97)$ & \\
30.6 (RI) & 1 & \\
45-50 (FC) & 1 & 0.983 \\
Type of primary surgery & $1.81(1.00-3.24)$ & \\
AR &
\end{tabular}

Values in parentheses are $95 \%$ confidence intervals. R0, microscopically radical; R1 microscopically irradical; R2, macroscopically irradical; EBRT, external beam radiation therapy; LRRC, locally recurrent rectal cancer; Gy, Gray; RI, re-irradiation; FC, full course radiotherapy; AR, anterior resection; APR, abdominoperineal resection.

the fear of toxicity. ${ }^{24,36}$ However, as confirmed by others, patients can undergo re-irradiation with an additional dose of 30-40 Gy with acceptable risks, provided that the small intestine is located outside the irradiation field and the interval between previous irradiation and re-irradiation is more than 6 months. ${ }^{10,26,37-39}$ As we found earlier, ${ }^{14}$ the intensive treatment brings along complications. However, it is difficult to find out if these complications are due to the tumor itself or due to the treatment. In the perspective of severe symptoms of untreated locally recurrent rectal cancer, the intensive multimodality treatment is justified.
TABLE 6. Factors influencing radicality of LRRC resection

\begin{tabular}{|c|c|c|c|}
\hline & Radical & Irradical & $P^{*}$ \\
\hline \multicolumn{4}{|l|}{ Gender } \\
\hline Male & $46(54.8)$ & $38(45.2)$ & \multirow[t]{2}{*}{0.614} \\
\hline Female & $38(60.3)$ & $25(39.7)$ & \\
\hline \multicolumn{4}{|l|}{ Age (years) } \\
\hline$<60$ & $30(54.5)$ & $25(45.5)$ & \multirow[t]{2}{*}{0.731} \\
\hline$\geq 60$ & $54(58.7)$ & $38(41.3)$ & \\
\hline \multicolumn{4}{|l|}{ Interval } \\
\hline$\leq 2$ years & $42(61.8)$ & $26(38.2)$ & \multirow[t]{2}{*}{0.319} \\
\hline$>2$ years & $42(53.2)$ & $37(46.8)$ & \\
\hline \multicolumn{4}{|l|}{ Primary TNM stage } \\
\hline I & $11(64.7)$ & $6(35.3)$ & \multirow[t]{3}{*}{0.189} \\
\hline II & $43(63.2)$ & $25(36.8)$ & \\
\hline III & $29(48.3)$ & $31(51.7)$ & \\
\hline \multicolumn{4}{|l|}{ Type of primary surgery } \\
\hline AR & $61(62.2)$ & $37(37.8)$ & \multirow[t]{2}{*}{0.044} \\
\hline APR & $19(43.2)$ & $25(56.8)$ & \\
\hline \multicolumn{4}{|l|}{ Previous EBRT (Gy) } \\
\hline 0 & $41(59.4)$ & $28(40.6)$ & \multirow[t]{3}{*}{0.232} \\
\hline 25 (short course) & $20(66.7)$ & $10(33.3)$ & \\
\hline 45-50 (long course) & $23(47.9)$ & $25(52.1)$ & \\
\hline \multicolumn{4}{|l|}{ EBRT for LRRC (Gy) } \\
\hline No & $7(29.2)$ & $17(70.8)$ & \multirow[t]{3}{*}{0.009} \\
\hline 30.6 (re-irradiation) & $37(64.9)$ & $20(35.1)$ & \\
\hline 45-50 (full course) & $40(60.6)$ & $26(39.4)$ & \\
\hline \multicolumn{4}{|l|}{ Radio(chemo) therapy } \\
\hline No & $7(29.2)$ & $17(70.8)$ & \multirow{3}{*}{0.008} \\
\hline Radiotherapy & $21(58.3)$ & $15(41.7)$ & \\
\hline Radiochemotherapy & $56(64.4)$ & $31(35.6)$ & \\
\hline \multicolumn{4}{|l|}{ Symptoms at diagnosis } \\
\hline So & $24(60.0)$ & $16(40.0)$ & \multirow[t]{3}{*}{0.030} \\
\hline $\mathrm{S} 1$ & $33(70.2)$ & $14(29.8)$ & \\
\hline S2 & $26(44.8)$ & $32(55.2)$ & \\
\hline
\end{tabular}

Values in parentheses are percentages. ${ }^{*} \chi^{2}$ test. LRRC, locally recurrent rectal cancer; AR, anterior resection; APR, abdominoperineal resection EBRT, external beam radiation therapy; Gy, Gray; LRRC, locally recurrent rectal cancer; S0, asymptomatic; S1, symptomatic without pain; S2, symptomatic with pain.

Other studies found a benefit of the addition of chemotherapy to the neoadjuvant radiotherapy. ${ }^{26,40}$ However, no statistically significant difference between radiotherapy and radiochemotherapy was demonstrated in our study. This might be due to small patient numbers.

Furthermore, higher TNM stage of the primary rectal cancer was an important negative prognostic factor. It did not influence local control, but was a significant predictor of OS and MFS on both univariate and multivariable analysis. These findings suggest that positive lymph nodes are representative of further metastatic spread of the disease at the time of primary tumor resection. Vermaas et al. ${ }^{30}$ and Hruby et al. ${ }^{41}$ reported similar results. Primary nodepositive disease resulted in a significant worse prognosis $(P=0.0009$ and $P<0.001$, respectively). This might be an indication to deliver adjuvant chemotherapy after LRRC resection in order to control or prevent systemic disease. 
Other publications showed only a trend towards improved survival with a lower primary TNM stage $^{11,37}$ or no relation at all. ${ }^{18,21,25,40,42}$

Not all LRRC patients described in the literature can be compared to those studied in this paper. For example, van den Brink et al. ${ }^{11}$ described patients from the Dutch TME trial who developed a LRRC. They found an early metastasis rate that approximated $75 \%$, with $40 \%$ of these patients already having a distant metastasis at time of LRRC diagnosis. In contrast, the patients in this study were already screened for concomitant metastases before they were referred to our hospital. Therefore, it is not surprising that the number of early metastases was lower in this study. Despite the favorable selection, $50 \%$ of these patients still developed metastases over time (Fig. 3). The multimodality treatment used focuses primarily on local control. The relative high rate of systemic disease may be an argument to include systemic treatment in the multimodality strategy. If systemic treatment would be administered in a neoadjuvant fashion, response to this treatment might be used as a selection criterion for further invasive procedures: local responders not developing metastases may be better candidates for extended surgery, whereas those who show local progression or even develop metastases under chemotherapy could be spared unnecessary extended surgery. The intensive multimodality treatment is not intended to compensate for macroscopically residual tumor. Therefore, a R2 resection may be considered as a treatment failure. However, at present there is still no modality to discriminate reliably between tissue fibrosis with and without tumor cells. Excluding all patients with severe fibrosis would mean that a proportion of these patients would have been denied a potentially curative resection. Therefore, it is impossible to avoid R2 resections, given the limitations of preoperative staging.

Some comments can be made about this study. It was a retrospective analysis, but the data have been collected prospectively. Heterogeneity in terms of neoadjuvant treatment was due to changes in treatment perspectives (e.g., introduction of total mesorectal excision and preoperative radio(chemo-) therapy in primary rectal cancer treatment) in this long period of patient accrual. For example in the early years of the study, re-irradiation was not considered to be a treatment option, but because of more evidence of the feasibility of this approach in later years, ${ }^{39}$ it was started in 1997. Furthermore, the benefit or advantage of IORT could not be discussed in our study as no comparison was possible between patients who received IORT and those who did not, since nearly all patients received IORT (and those who did not had specific reasons for it). Hashiguchi and co-workers ${ }^{43}$ found a significant survival benefit in patients who received IORT compared to patients who did not (3-year survival rate of $43 \%$ versus $5 \%$, $P=0.0007)$. This was also confirmed on multivariate analysis. Several studies describe an increase of the 5 -year survival rate of $15 \%$ or more after the introduction of IORT as a component of multimodality treatment. ${ }^{32,44,45}$ However, the role of IORT remains debatable. ${ }^{29,46}$ Careful delivery of IORT is important. The ureters, blood vessels, soft tissue, bone, and pelvic nerves are structures at risk of injury from IORT. ${ }^{13,44,47,48}$ IOERT doses of 10-20 Gy are the maximum tolerable doses when combined with EBRT. Data from MGH showed that for primary rectal cancer the combined use of high-dose preoperative EBRT, surgical resection, and IOERT was tolerated well. ${ }^{13}$ However, in locally advanced recurrent disease the complication rate was higher. Nevertheless, Krempien et al. ${ }^{49}$ described very low long-term complications in patients with locally advanced primary rectal cancer treated with TME, IOERT, and pre- or postoperative EBRT. The 5- and 10 -year actuarial incidences of $\geq$ grade 3 late complications for the entire group were $9 \%$ and $13 \%$, respectively. IORT in combination with re-irradiation leads to a high radiobiological dose and our data support the assumption that this dose can be applied safely. However, large prospective randomized controlled studies are lacking.

As local re-recurrence is a great problem after treatment for local recurrence, the administration of high tumorcidal irradiation doses at the areas of risk follows a sound radiobiological principle. The potential contribution of IORT to the improved cure rates reported by others has also been achieved in this analysis.

In summary, in this patient population with a generally poor prognosis, the use of multimodality treatment and radical resection has resulted in a more favorable prognosis. Due to the rarity of this entity and the inability to perform randomized studies, the additional values of radiochemotherapy (versus radiotherapy), re-irradiation, and IORT (versus no IORT) were not clearly identifiable. Further evaluation is required. However, high rates of radical resections have been reached, leading to a significantly improved survival in patients with LRRC. Unfortunately, development of metastatic disease remains a great problem. In the future, attention has to be paid to better patient selection and appropriate 
systemic treatment in order to ameliorate prognosis even further.

\section{OPEN ACCESS}

This article is distributed under the terms of the Creative Commons Attribution Noncommercial License which permits any noncommercial use, distribution, and reproduction in any medium, provided the original author(s) and source are credited.

\section{REFERENCES}

1. Kapiteijn E, Marijnen CA, Nagtegaal ID, et al. Preoperative radiotherapy combined with total mesorectal excision for resectable rectal cancer. $N$ Engl $J$ Med 2001; 345:638-46.

2. Havenga K, Enker WE, Norstein J, et al. Improved survival and local control after total mesorectal excision or D3 lymphadenectomy in the treatment of primary rectal cancer: an international analysis of 1411 patients. Eur J Surg Oncol 1999; 25:368-74

3. Palmer G, Martling A, Cedermark B, et al. A populationbased study on the management and outcome in patients with locally recurrent rectal cancer. Ann Surg Oncol 2007; 14:44754.

4. Folkesson J, Birgisson H, Pahlman L, et al. Swedish Rectal Cancer Trial: long lasting benefits from radiotherapy on survival and local recurrence rate. J Clin Oncol 2005; 23:5644-50.

5. Wiggers T, van de Velde CH. Reduction "by half". The need for standardised surgical technique in studies of radiotherapy for rectal cancer. Eur J Surg 1999; 165:407-9.

6. Quirke P, Sebag-Montefiore D, Steele R, et al. Local recurrence after rectal cancer resection is strongly related to the plane of surgical dissection and is further reduced by preoperative short course radiotherapy. Preliminary results of the Medical Research Council (MRC) CR07 trial. J Clin Oncol 2006; 24:18s (suppl: abstr 3512).

7. Saito N, Koda K, Takiguchi N, et al. Curative surgery for local pelvic recurrence of rectal cancer. Dig Surg 2003; 20:192-9.

8. Temple WJ, Saettler EB. Locally recurrent rectal cancer: role of composite resection of extensive pelvic tumors with strategies for minimizing risk of recurrence. J Surg Oncol 2000; 73:47-58.

9. Wanebo HJ, Antoniuk P, Koness RJ, et al. Pelvic resection of recurrent rectal cancer: technical considerations and outcomes. Dis Colon Rectum 1999; 42:1438-48.

10. Glimelius B. Recurrent rectal cancer. The pre-irradiated primary tumour: can more radiotherapy be given? Colorectal Dis 2003; 5:501-3.

11. van den Brink M, Stiggelbout AM, van den Hout WB, et al. Clinical nature and prognosis of locally recurrent rectal cancer after total mesorectal excision with or without preoperative radiotherapy. J Clin Oncol 2004; 22:3958-64.

12. Willett CG, Czito BG, Tyler DS. Intraoperative radiation therapy. J Clin Oncol 2007; 25:971-7.

13. Willett CG. Intraoperative radiation therapy. Int $J$ Clin Oncol 2001; 6:209-14.

14. Mannaerts GH, Martijn H, Crommelin MA, et al. Intraoperative electron beam radiation therapy for locally recurrent rectal carcinoma. Int J Radiat Oncol Biol Phys 1999; 45:297-308.

15. Radice E, Dozois RR. Locally recurrent rectal cancer. Dig Surg 2001; 18:355-62.

16. Moriya Y. Treatment strategy for locally recurrent rectal cancer. Jpn J Clin Oncol 2006; 36:127-31.
17. Saito N, Koda K, Takiguchi N, et al. Surgery for local pelvic recurrence after resection of rectal cancer. Int $J$ Colorectal Dis $1998 ; 13: 32-8$.

18. Bakx R, van TH, van Lanschot JJ, et al. Surgical treatment of locally recurrent rectal cancer. Eur J Surg Oncol 2004; 30:85763.

19. Eble MJ, Lehnert T, Treiber M, et al. Moderate dose intraoperative and external beam radiotherapy for locally recurrent rectal carcinoma. Radiother Oncol 1998; 49:169-74.

20. Moriya Y, Akasu T, Fujita S, et al. Total pelvic exenteration with distal sacrectomy for fixed recurrent rectal cancer in the pelvis. Dis Colon Rectum 2004; 47:2047-53.

21. Shoup M, Guillem JG, Alektiar KM, et al. Predictors of survival in recurrent rectal cancer after resection and intraoperative radiotherapy. Dis Colon Rectum 2002; 45:585-92.

22. Wells BJ, Stotland P, Ko MA, et al. Results of an aggressive approach to resection of locally recurrent rectal cancer. Ann Surg Oncol 2007; 14:390-5.

23. Wiggers T, de Vries MR, Veeze-Kuypers B. Surgery for local recurrence of rectal carcinoma. Dis Colon Rectum 1996; 39:323-8.

24. Rodel C, Grabenbauer GG, Matzel KE, et al. Extensive surgery after high-dose preoperative chemoradiotherapy for locally advanced recurrent rectal cancer. Dis Colon Rectum 2000; 43:312-9.

25. Asoglu O, Karanlik H, Muslumanoglu M, et al. Prognostic and predictive factors after surgical treatment for locally recurrent rectal cancer: a single institute experience. Eur J Surg Oncol 2007; 33(10):1199-206.

26. Bedrosian I, Giacco G, Pederson L, et al. Outcome after curative resection for locally recurrent rectal cancer. Dis Colon Rectum 2006; 49:175-82.

27. Melton GB, Paty PB, Boland PJ, et al. Sacral resection for recurrent rectal cancer: analysis of morbidity and treatment results. Dis Colon Rectum 2006; 49:1099-107.

28. Lopez-Kostner F, Fazio VW, Vignali A, et al. Locally recurrent rectal cancer: predictors and success of salvage surgery. Dis Colon Rectum 2001; 44:173-8.

29. Wiig JN, Tveit KM, Poulsen JP, et al. Preoperative irradiation and surgery for recurrent rectal cancer. Will intraoperative radiotherapy (IORT) be of additional benefit? A prospective study. Radiother Oncol 2002; 62:207-13.

30. Vermaas M, Ferenschild FT, Nuyttens JJ, et al. Preoperative radiotherapy improves outcome in recurrent rectal cancer. Dis Colon Rectum 2005; 48:918-28.

31. Suzuki K, Dozois RR, Devine RM, et al. Curative reoperations for locally recurrent rectal cancer. Dis Colon Rectum 1996; 39:730-6.

32. Suzuki K, Gunderson LL, Devine RM, et al. Intraoperative irradiation after palliative surgery for locally recurrent rectal cancer. Cancer 1995; 75:939-52.

33. Boyle KM, Sagar PM, Chalmers AG, et al. Surgery for locally recurrent rectal cancer. Dis Colon Rectum 2005; 48:929-37.

34. Ogunbiyi OA, McKenna K, Birnbaum EH, et al. Aggressive surgical management of recurrent rectal cancer-is it worthwhile? Dis Colon Rectum 1997; 40:150-5.

35. Bergamaschi R, Pessaux P, Burtin P, et al. Abdominoperineal resection for locally recurrent rectal cancer. Tech Coloproctol 2001; 5:97-102.

36. Vermaas M, Ferenschild FT, Verhoef C, et al. Total pelvic exenteration for primary locally advanced and locally recurrent rectal cancer. Eur J Surg Oncol 2007; 33:452-8.

37. Mohiuddin M, Marks G, Marks J. Long-term results of reirradiation for patients with recurrent rectal carcinoma. Cancer 2002; 95:1144-50.

38. Valentini V, Morganti AG, Gambacorta MA, et al. Preoperative hyperfractionated chemoradiation for locally recurrent rectal cancer in patients previously irradiated to the pelvis: A multicentric phase II study. Int J Radiat Oncol Biol Phys 2006; 64:1129-39. 
39. Mohiuddin M, Marks GM, Lingareddy V, et al. Curative surgical resection following reirradiation for recurrent rectal cancer. Int J Radiat Oncol Biol Phys 1997; 39:643-9.

40. Caricato M, Borzomati D, Ausania F, et al. Prognostic factors after surgery for locally recurrent rectal cancer: an overview. Eur J Surg Oncol 2006; 32:126-32.

41. Hruby G, Barton M, Miles S, et al. Sites of local recurrence after surgery, with or without chemotherapy, for rectal cancer: implications for radiotherapy field design. Int $J$ Radiat Oncol Biol Phys 2003; 55:138-43.

42. Salo JC, Paty PB, Guillem J, et al. Surgical salvage of recurrent rectal carcinoma after curative resection: a 10-year experience. Ann Surg Oncol 1999; 6:171-7.

43. Hashiguchi Y, Sekine T, Sakamoto H, et al. Intraoperative irradiation after surgery for locally recurrent rectal cancer. $D i$ Colon Rectum 1999; 42:886-93.

44. Lindel K, Willett CG, Shellito PC, et al. Intraoperative radiation therapy for locally advanced recurrent rectal or rectosigmoid cancer. Radiother Oncol 2001; 58:83-7.
45. Mannaerts GH, Rutten HJ, Martijn H, et al. Comparison of intraoperative radiation therapy-containing multimodality treatment with historical treatment modalities for locally recurrent rectal cancer. Dis Colon Rectum 2001; 44:1749-58.

46. Wiig JN, Poulsen JP, Tveit KM, et al. Intra-operative irradiation (IORT) for primary advanced and recurrent rectal cancer. A need for randomised studies. Eur J Cancer 2000; 36: 868-74.

47. Haddock MG, Gunderson LL, Nelson H, et al. Intraoperative irradiation for locally recurrent colorectal cancer in previously irradiated patients. Int J Radiat Oncol Biol Phys 2001; 49:126774

48. Heriot AG, Tekkis PP, Darzi A, et al. Surgery for local recurrence of rectal cancer. Colorectal Dis 2006; 8:733-47.

49. Krempien R, Roeder F, Oertel S, et al. Long-term results of intraoperative presacral electron boost radiotherapy (IOERT) in combination with total mesorectal excision (TME) and chemoradiation in patients with locally advanced rectal cancer. Int J Radiat Oncol Biol Phys 2006; 66:1143-51. 